\title{
Vitamin C alleviates aging defects in a stem cell model for Werner syndrome
}

\author{
Ying $\mathrm{Li}^{1,2}$, Weizhou Zhang ${ }^{5}$, Liang Chang ${ }^{4}$, Yan $\mathrm{Han}^{1,2}$, Liang Sun ${ }^{6}$, Xiaojun Gong ${ }^{9}$, Hong Tang ${ }^{9}$, \\ Zunpeng Liu ${ }^{1,2}$, Huichao Deng ${ }^{1,2}$, Yanxia Ye ${ }^{3}$, Yu Wang ${ }^{3}$, Jian $\mathrm{Li}^{6}$, Jie Qiao ${ }^{4}$, Jing Qu ${ }^{2,3 凶}$, Weiqi Zhang ${ }^{1,7 \bowtie}$, \\ Guang-Hui Liu ${ }^{1,2,7,8}$ \\ ${ }^{1}$ National Laboratory of Biomacromolecules, CAS Center for Excellence in Biomacromolecules Institute of Biophysics, \\ Chinese Academy of Sciences, Beijing 100101, China \\ 2 University of Chinese Academy of Sciences, Beijing 100049, China \\ ${ }^{3}$ State Key Laboratory of Stem Cell and Reproductive Biology, Institute of Zoology, Chinese Academy of Sciences, \\ Beijing 100101, China \\ ${ }^{4}$ Department of Gynecology and Obstetrics, Peking University Third Hospital, Beijing 100191, China \\ ${ }^{5}$ Department of Pathology, Carver College of Medicine, University of lowa, lowa City, IA 52242, USA \\ ${ }^{6}$ The Key Laboratory of Geriatrics, Beijing Hospital \& Beijing Institute of Geriatrics, Ministry of Health, Beijing 100730, \\ China \\ ${ }^{7}$ FSU-CAS Innovation Institute, Foshan University, Foshan 528000, China \\ ${ }^{8}$ Beijing Institute for Brain Disorders, Beijing 100069, China \\ ${ }^{9}$ Department of Pediatrics, Beijing Shijitan Hospital Capital Medical University, Peking University Ninth School \\ of Clinical Medicine, Beijing 100038, China \\ $\triangle$ Correspondence: qujing@ioz.ac.cn (J. Qu), weiqizhang@aliyun.com (W. Zhang), ghliu@ibp.ac.cn (G.-H. Liu) \\ Received March 3, 2016 Accepted April 29, 2016
}

\begin{abstract}
Werner syndrome (WS) is a premature aging disorder that mainly affects tissues derived from mesoderm. We have recently developed a novel human WS model using WRN-deficient human mesenchymal stem cells (MSCs). This model recapitulates many phenotypic features of WS. Based on a screen of a number of chemicals, here we found that Vitamin $C$ exerts most efficient rescue for many features in premature aging as shown in WRN-deficient MSCs, including cell growth arrest, increased reactive oxygen species levels, telomere attrition, excessive secretion of inflammatory factors, as well as disorganization of nuclear lamina and heterochromatin. Moreover, Vitamin $C$ restores in vivo viability of MSCs in a mouse model. RNA sequencing analysis indicates that Vitamin C
\end{abstract}

Ying Li, Weizhou Zhang, and Liang Chang have contributed equally to this work

Electronic supplementary material The online version of this article (doi:10.1007/s13238-016-0278-1) contains supplementary material, which is available to authorized users. alters the expression of a series of genes involved in chromatin condensation, cell cycle regulation, DNA replication, and DNA damage repair pathways in WRNdeficient MSCs. Our results identify Vitamin $C$ as a rejuvenating factor for WS MSCs, which holds the potential of being applied as a novel type of treatment of WS.

KEYWORDS Vitamin C, stem cell, aging, Werner syndrome

\section{INTRODUCTION}

Aging is defined as a time-dependent deterioration of organism's physiological functions that leads to loss of homeostasis and consequently increases susceptibility to morbidity and mortality (Benayoun et al., 2015; Burtner and Kennedy, 2010; Campisi, 2013; Kudlow et al., 2007; LopezOtin et al., 2013). Werner Syndrome (referred to as WS, also known as adult progeria) is a premature aging disorder with phenotypes such as grey hair, osteoporosis, diabetes, and cancer. WS is caused by mutations in the WRN gene, which is involved in several fundamental cellular mechanisms, including DNA replication, DNA repair, and telomere 


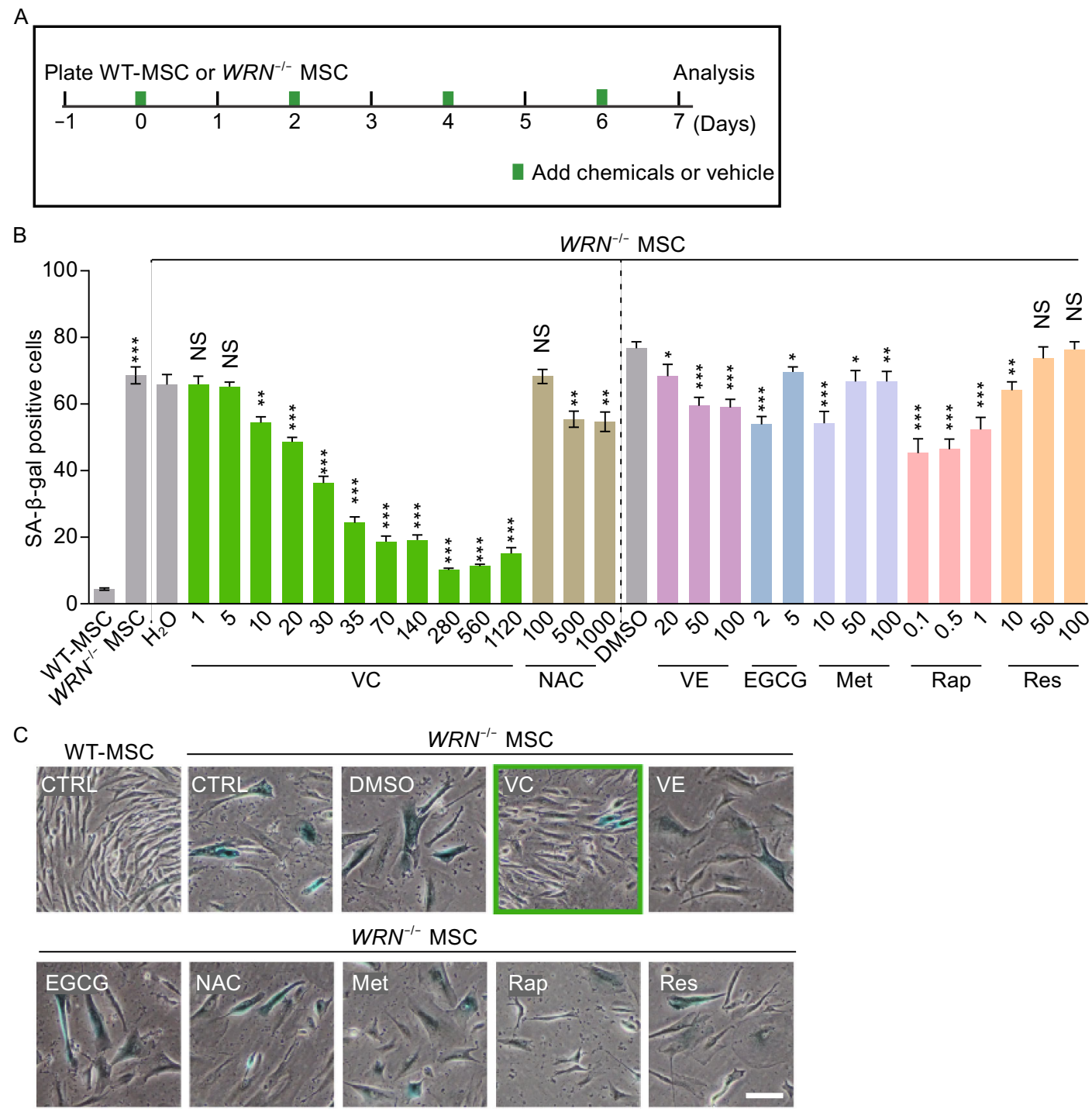

Figure 1. Chemicals screening for alleviating premature aging in WS MSCs. (A) Schematic demonstration of the chemical screening protocol. $3 \times 10^{4}$ wild-type (WT) or WRN ${ }^{-1}$ MSCs (P5) were seeded in one well of 6-well dish, following with the treatment with the chemicals or vehicle control (water or DMSO) and refreshed every other day. Cell senescence, assayed by senescence associated $\beta$-galactosidase (SA- $\beta$-gal) staining, was analyzed 1 week later. (B) Frequency of SA- $\beta$-gal positive cells in WT or WRN ${ }^{\prime-}$ MSCs with or without chemical treatment. For each molecule, different concentrations were used as indicated along the $X$ axis. Data are represented as mean \pm SEM, ${ }^{*} P<0.05,{ }^{* *} P<0.01$, ${ }^{* \star *} P<0.001$, NS, not significant by $t$ test; $n \geq 3$. (C) Representative images of SA- $\beta$-gal staining. VC: $280 \mu \mathrm{mol} / \mathrm{L}$; VE: $20 \mu \mathrm{mol} / \mathrm{L}$; EGCG: $2 \mu \mathrm{mol} / \mathrm{L}$; NAC: $100 \mu \mathrm{mol} / \mathrm{L}$; Met: $10 \mu \mathrm{mol} / \mathrm{L}$; Rap: $0.1 \mu \mathrm{mol} / \mathrm{L} ; \mathrm{Res}: 10$ $\mu \mathrm{mol} / \mathrm{L}$. Scale bar, $100 \mu \mathrm{m}$.

maintenance (Burtner and Kennedy, 2010; Kudlow et al., 2007; Lopez-Otin et al., 2013). Since the expression of WRN also decreases during physiological aging (Polosak et al., 2011; Zhang et al., 2015), WS may be a relevant model for studying physiological aging and aging-associated disorders (Burtner and Kennedy, 2010; Kudlow et al., 2007; LopezOtin et al., 2013).

The advances in pluripotent stem cell and gene editing techniques have opened a new avenue to study the pathogenesis of human premature aging syndromes and aging-related diseases (Fu et al., 2016; Liu et al., 2011; Liu et al., 2012b; Liu et al., 2014; Lo Cicero and Nissan, 2015; Miller et al., 2013; Pan et al., 2016; Zhang et al., 2015). They also provide a powerful platform for drug screening and validation of their efficacy (Blondel et al., 2016; Liu et al., 2012a; Liu et al., 2012b; Liu et al., 2014; Yang et al., 2014; Zhang et al., 2013). We have recently developed a human stem cell model by homozygous depletion of the 
A

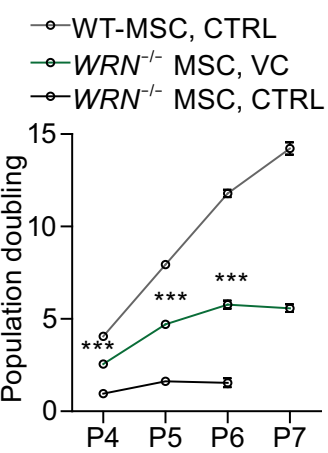

B

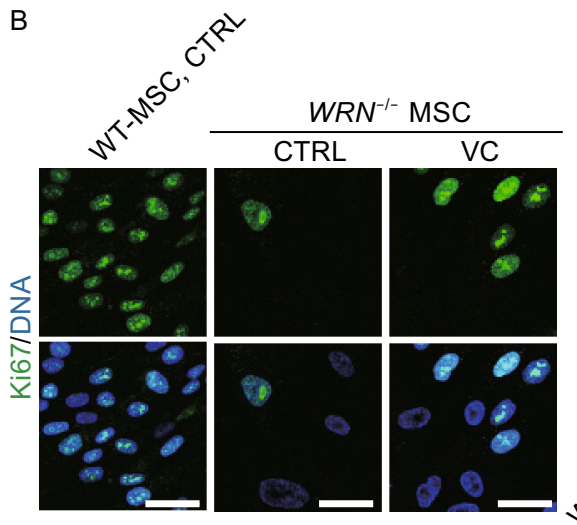

$E$

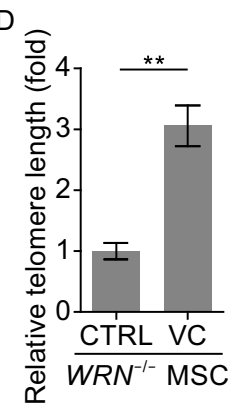

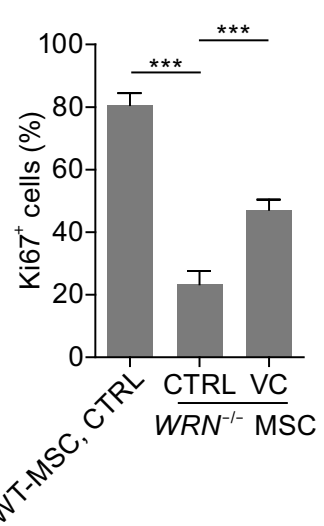

C

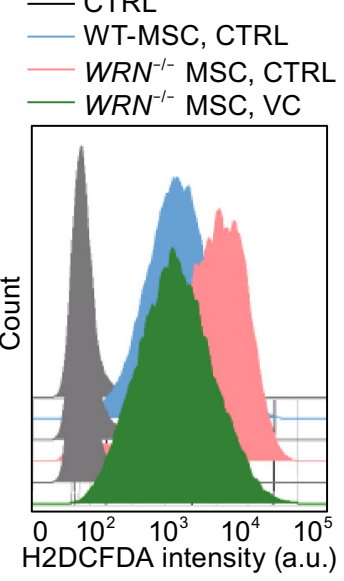

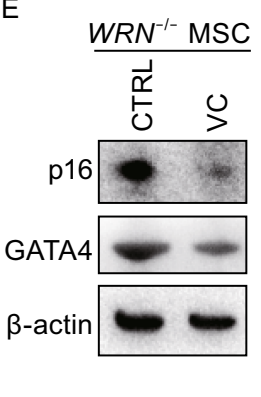
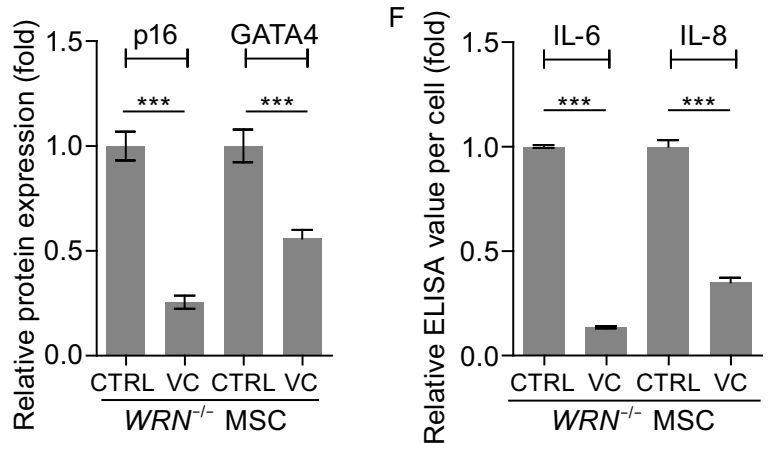

Figure 2. VC represses aging properties in WS MSCs. (A) Growth curve analyzing the population doubling of MSCs. (B) VC promoted proliferation in $W R N^{--}$MSCs. Representative immunofluorescence staining (left) and quantitative analysis (right) of Ki67 in vehicle or VC treated (7 days) WT MSCs and WRN ${ }^{-1}$ MSCs. Scale bar, $50 \mu \mathrm{m}$. (C) H2DCFDA based measurement of reactive oxygen species (ROS) in vehicle or VC treated (7 days) WT MSCs and WRN ${ }^{1-}$ MSCs. (D) Telomere lengths of WS MSCs with or without VC treatment were measured by quantitative RT-PCR. (E) Expression of senescence-associated proteins p16 ${ }^{\text {Ink4a }}$ and GATA4 was examined by Western blot, and quantitative results were shown on the right. (F) ELISA showing a decrease in IL-6 and IL-8 secretion in $W R N^{\prime-}$ MSCs after VC treatment. The values were normalized by the cell numbers. Data are represented as mean $\pm \mathrm{SEM}$, ${ }^{\star \star} P<$ $0.01,{ }^{* * *} P<0.001 ; n \geq 3$.

exons 15 and 16 of $W R N$ alleles, which recapitulates the major cellular defects of WS, including accelerated senescence, growth arrest, telomere attrition, increased DNA damage response, excessive production of inflammatory factors, as well as increased stem cell attrition in the in vivo niche (Zhang et al., 2015). We also identify heterochromatin disorganization as a driver for WS MSC aging, and overexpression of heterochromatin component HP1 $\alpha$ can partially rescue the accelerated aging defects in the WS MSCs (Zhang et al., 2015). These findings suggest that epigenetic alterations could underlie human cellular aging, and the "epigenetic aging" can be repressed or reversed under specific context. It is unknown, however, if the premature aging processes can be alleviated by chemicals or drugs.

Here, utilizing the WS MSC model, we tested the potential rescuing effect with a group of compounds which have been reported with "anti-aging" or "longevity-promoting" activity from different model organisms. Among them, Vitamin C
(VC, also known as ascorbic acid) showed the best efficacy on alleviation of the aging defects in WS MSCs.

\section{RESULTS}

Screening for chemicals capable of repressing accelerated cellular senescence in WS MSCs

Using our recently established WS MSC aging model (Zhang et al., 2015), we have screened a panel of known anti-oxidants and other chemicals with reported anti-aging effects, including VC, Vitamin E (VE), (-)-epigallocatechin gallate (EGCG), N-Actyl-L-cysteine (NAC), Metformin (Met), Rapamycin (Rap), and Resveratrol (Res) (Baur et al., 2006; Cao et al., 2011; Dallaire et al., 2014; Harrison et al., 2009; La Fata et al., 2014; Lebel et al., 2010; Martin-Montalvo et al., 2013; Na et al., 2008). We designed an in vitro screening platform by using late passage (passage 5) of $W R N^{-1}$ MSCs at low confluence. The low cell density allowed for evaluating 

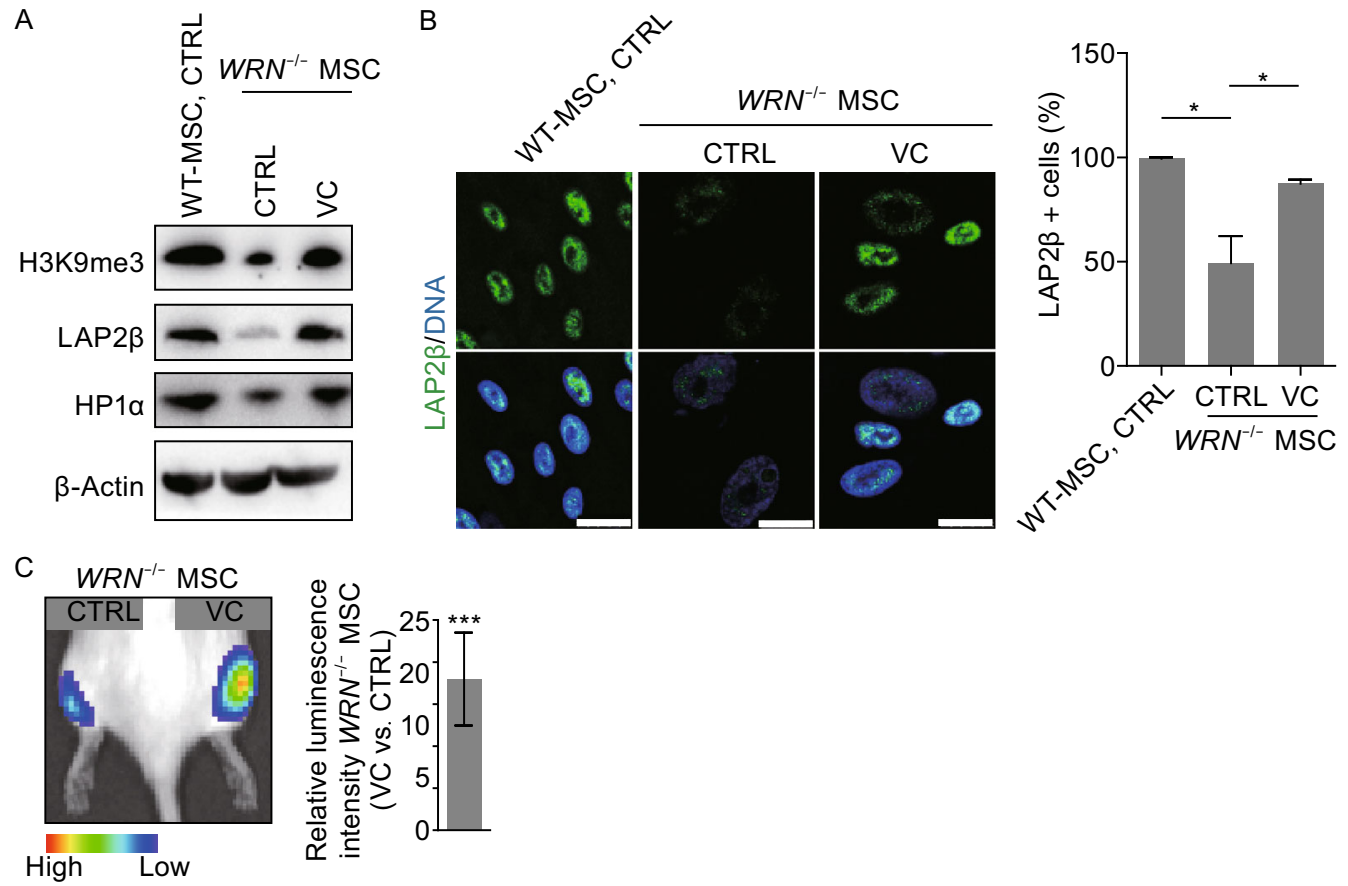

Figure 3. VC restores epigenetic parameters and in vivo viability of WS MSCs. (A) Western blot analysis of the indicated proteins in MSCs. (B) VC increased heterochromatin markers by immunofluorescence staining. Representative immunofluorescence staining (left) and quantitative analysis (right) of LAP2 $\beta$ in vehicle or VC treated (7 days) WT MSCs and WRN ${ }^{\prime-}$ MSCs. Scale bar, 25 $\mu \mathrm{m}$. (C) Luciferase activity of WS MSCs was detected by in vivo imaging system (IVIS) one week after implantation, and quantitative results were shown on the right. All data are represented as mean \pm SEM. ${ }^{*} P<0.05$, ${ }^{\star \star \star} P<0.001$ by $t$ test; $n \geq 3$.

stringent phenotypic rescue and maximizing the efficacy of chemicals. We treated the cells with the indicated chemicals up to one week (Fig. 1A). Among all the chemicals included, we identified $\mathrm{VC}$ as the most potent agent that significantly reduced the frequency of senescence-associated- $\beta$-galactosidase (SA- $\beta$-gal) positive cells in a dose-dependent manner (Fig. 1B and 1C). The efficacy of $\mathrm{VC}$ on suppressing senescence was very prominent at physiological concentrations starting from as low as $10 \mu \mathrm{mol} / \mathrm{L}$, relative to the vehicle treatment group. At the proposed physiological doses of plasma VC (Du et al., 2012), ranging from 35 to $70 \mu \mathrm{mol} / \mathrm{L}, \mathrm{VC}$ exhibited close to the optimal suppressive effect on senescence (compared between $68 \%$ in control group, $20 \%-23 \%$ in physiological dosages (35-70 $\mu \mathrm{mol} / \mathrm{L})$, and $10 \%$ in optimal dose $(280-560 \mu \mathrm{mol} / \mathrm{L})$ (Fig. 1B). Other compounds, including VE, EGCG, NAC, Met, Rap, and Res also showed mild activity in reducing SA- $\beta$-gal positive subpopulation (Fig. 1B and 1C), but the effects were not as significant as VC's. These results indicate that VC has a unique activity in repressing accelerated senescence in WS MSCs.

\section{VC suppresses aging-related parameters}

We have shown that the WS MSCs exhibited many features of premature cellular senescence, such as decreased proliferation, elevated senescence-associated secretory phenotype (SASP), and heterochromatin alterations etc. (Zhang et al., 2015). To determine the impact of VC on these parameters, we treated WS MSCs with VC and examined different cellular properties related to aging. Consistent with the reduced premature cellular senescence, VC treatment reactivated the cellular proliferation potential and upregulated the frequency of Ki67 positive cells (Fig. 2A and 2B). Reactive oxygen species (ROS) production, as indicated by the positive staining of H2DCFDA, was significantly elevated in the senescent WS MSCs as previously reported for WS mouse fibroblasts (Labbe et al., 2010), which was reduced to a similar level as in the WT MSC cells by VC treatment (Fig. 2C). In addition, treatment of WS MSCs with VC repressed accelerated telomere shortening (Fig. 2D) (Zhang et al., 2015), down-regulated expression of aging markers, such as p16 $6^{\text {Ink4a }}$ and GATA4 (Fig. 2E) (Kang et al., 2015), and effectively alleviated SASP, including the production of pro-inflammatory cytokines such as IL-6 and IL-8 (Fig. 2F).

Heterochromatin alteration is one of the hallmarks and is a driver for WS MSC aging (Lopez-Otin et al., 2013; Zhang et al., 2015). Western blotting showed that levels of heterochromatin markers HP1 $\alpha$ and H3K9me 3 were up-regulated upon VC treatment, indicating that VC promotes remodeling of heterochromatin to a younger state. In line with the heterochromatin changes, the expression of LAP $2 \beta$, the heterochromatin-anchoring inner nuclear membrane protein, was also elevated (Fig. $3 \mathrm{~A}$ and $3 \mathrm{~B}$ ). In addition, we 
found an increase in the number of nuclear foci for $\mathrm{Y}-\mathrm{H} 2 \mathrm{AX}$ and phosphorylated ATM/ATR substrates in WRN-deficient MSCs; VC had no influence on these foci formation (Fig. S1A), suggesting a possibility that the restoration of heterochromatin and nuclear lamina components may not be associated with alleviation of DNA damage response (DDR) in WS MSCs. Together, these results indicate that VC is able to rejuvenate the heterochromatin and nuclear lamina architectures in WS MSCs, a process independent of the DNA damage response.

To investigate whether VC can restore the MSC's in vivo activity, luciferase-labeled WS MSCs were pre-treated with VC, and implanted into the tibialis anterior muscle of the immunodeficient mice, and then engraftment and survival were determined by measuring luminescence signals after 7 days. In line with the observed repression of accelerated cellular decay in vitro, VC treatment effectively restored the in vivo viability of WS MSCs (Fig. $3 C$ ).

\section{VC inhibits aging related genes and pathways in the WS MSC model}

To uncover the molecular mechanism underlying how VC rejuvenates WS MSCs, we performed genome-wide RNA sequencing (RNA-seq). We identified 1595 upregulated genes and 1419 downregulated genes ( $\mid \log _{2}$ (Fold change)| $>1, P$ value $<0.05)$ in $\mathrm{VC}$ treated WS MSCs relative to vehicle treated cells (Fig. 4A, S1B-C and Supplementary tables). Gene Ontology (GO) Term analysis for cellular component, biological pathways and molecular functions indicated that the most significant pathway $(P$ value $=0.00036)$ for the VC-upregulated genes in WS MSCs was "chromosome organization" (Fig. S1D), which is also the most significant GO term for downregulated genes in WS MSCs compared to wild-type MSCs (Zhang et al., 2015). Notably, VC induced the re-expression of chromosome-packaging proteins at centromeres in WS MSCs, which is consistent with a rescue of cellular aging process (Fig. S1D) (Zhang et al., 2015).

We also analyzed the differentially expressed genes using KEGG database. We found "cell cycle" as the top remarkable set of genes upregulated by VC, in agreement with the increased $\mathrm{Ki} 67$ staining as the proliferation index (Fig. 3B). Consistently, the transcripts for many of these cell cycle genes are also the ones we initially identified to be down-regulated in WS MSCs (Zhang et al., 2015). We noticed that the molecular network for cell cycle entry was partially re-activated by $V C$ (Fig. 4B and $4 C$ ). Of note, the cell mitosis-promoting proteins such as PCNA, CDK1, and CDK2 were also significantly upregulated in VC-treated WS MSCs (Fig. 4C and 4D). In addition, we also found that VC was involved in DNA replication and repair pathways. For example, VC treatment led to the reactivation of the DNA replication pathway that was repressed in WRN-deficient MSCs (Fig. 4B) (Zhang et al., 2015), and of the various DNA repair pathways including mismatch repair, homologous recombination, base excision repair, and Fanconi anemia pathway (Zhang et al., 2015) (Fig. 4B). On the other hand, VC down-regulated several signaling pathways that are normally activated during cellular senescence, such as P53, FoxO, and HIF-1 pathways (Lopez-Otin et al., 2013; Martins et al., 2015), as well as the pathways related to lysosome degradation and protein-processing in endoplasmic reticulum (ER) (Fig. 4B). These changes were validated by quantitative RT-PCR (Fig. 4D). Together, these results clearly indicate that VC plays a role in re-activation of agingsuppressing genes and related signaling pathways at the transcriptional level.

\section{DISCUSSION}

The combination of gene editing technique and human pluripotent stem cells (hPSC) including induced pluripotent stem cells (iPSCs) and embryonic stem cells offers a new way to model genetic diseases for studying disease-causing mechanisms and screening drugs in vitro. Since 2011, we and others have shown the capacity of hPSC disease models in recapitulating disease defects in various human genetic disorders including Hutchinson-Gliford progeria syndrome (HGPS), Parkinson's diseases, Fanconi Anemia, xeroderma pigmentosum, glioblastoma, and WS (Cheung et al., 2014; Duan et al., 2015; Fu et al., 2016; Liu et al., 2011; Liu et al., 2012a; Liu et al., 2012b; Liu et al., 2014; Park et al., 2008; Saha and Jaenisch, 2009; Yu et al., 2013; Zhang et al., 2015). We have also provided evidence showing that these disease models could be used to examine the functional effects of chemical compounds as well as to re-purpose the old drugs already approved for different diseases in clinics. In this study, we identify VC as a potent agent to alleviate aging process in a stem cell model for adult progeria. Among the compounds we tested, VC has the most significant effect on repressing cellular senescence (indicated by SA- $\beta$-gal staining) and on promoting the WS MSC growth. For example, whereas the mTOR inhibitor rapamycin has been reported to improve the proliferation potential in HGPS fibroblasts (Cao et al., 2011), it showed marked repressive effect on the self-renewal of WS MSCs (Fig. 1B, 1C and data not shown). VC effectively represses the accelerated cellular senescence, improves the stem cell self-renewal, decreases SASP in WS MSCs, and alleviates telomere attrition. This finding is of great interest because previous studies on $C$. elegans and mouse models have showed that a continuous long-term treatment of WS individuals with high doses of $\mathrm{VC}$ is a promising therapeutic approach for this syndrome (Dallaire et al., 2014; Lebel et al., 2010). Our study, together with evidences from C. elegans and mouse models, provides an important cue to WS therapy. Recent study indicated that the blood of WS patient has increased level of IL-6 (Davis and Kipling, 2006). Consistently, our WS stem cell model recapitulated the upregulation 


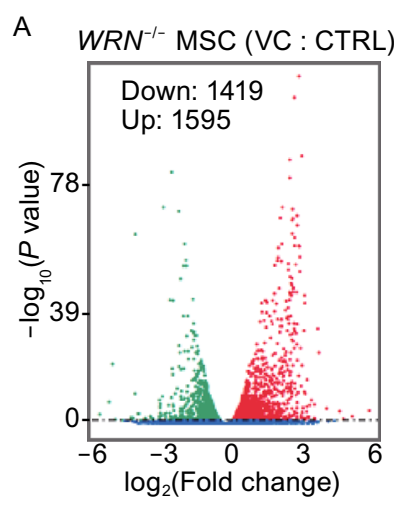

C
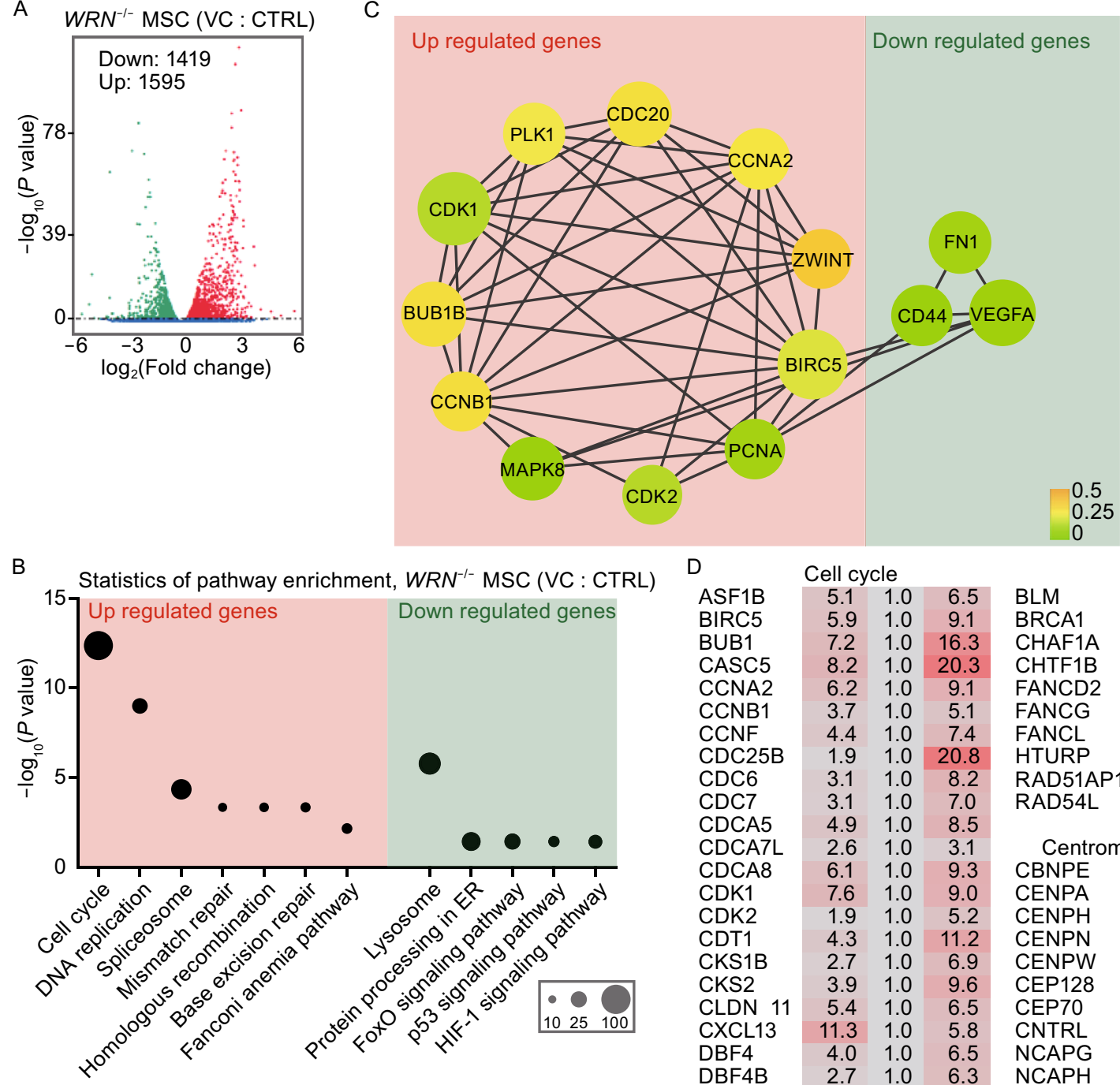

\begin{tabular}{lrrrl|r|r|r} 
D & \multicolumn{1}{c}{ Cell cycle } & \multicolumn{5}{c}{ DNA repair } \\
ASF1B & 5.1 & 1.0 & 6.5 & BLM & 8.2 & 1.0 & 16.7 \\
BIRC5 & 5.9 & 1.0 & 9.1 & BRCA1 & 6.8 & 1.0 & 6.3 \\
BUB1 & 7.2 & 1.0 & 16.3 & CHAF1A & 3.0 & 1.0 & 6.5 \\
CASC5 & 8.2 & 1.0 & 20.3 & CHTF1B & 2.8 & 1.0 & 4.2 \\
CCNA2 & 6.2 & 1.0 & 9.1 & FANCD2 & 3.7 & 1.0 & 10.1 \\
CCNB1 & 3.7 & 1.0 & 5.1 & FANCG & 7.9 & 1.0 & 7.6 \\
CCNF & 4.4 & 1.0 & 7.4 & FANCL & 5.2 & 1.0 & 8.2 \\
CDC25B & 1.9 & 1.0 & 20.8 & HTURP & 13.5 & 1.0 & 10.6 \\
CDC6 & 3.1 & 1.0 & 8.2 & RAD51AP1 & 6.1 & 1.0 & 12.8 \\
CDC7 & 3.1 & 1.0 & 7.0 & RAD54L & 9.5 & 1.0 & 8.7
\end{tabular}

$\begin{array}{llll}\text { CDCA5 } & 4.9 & 1.0 & 8.5 \\ \text { CDCA7L } & 2.6 & 1.0 & 3.1\end{array}$

Centromere-related proteins \begin{tabular}{llllllll} 
CDCA8 & 2.6 & 1.0 & 3.1 & \multicolumn{3}{c}{ Centromere-related proteins } \\
\hline & 6.1 & 1.0 & 9.3 & CBNPE & 5.5 & 1.0 & 6.8
\end{tabular} $\begin{array}{lllllllll}\text { CDK1 } & 7.6 & 1.0 & 9.0 & \text { CENPA } & 8.1 & 1.0 & 17.1\end{array}$ \begin{tabular}{l|rrr|r|rrr} 
CDK2 & 1.9 & 1.0 & 5.2 & CENPH & 11.7 & 1.0 & 16.8
\end{tabular} \begin{tabular}{l|rrr|r|r|r|r|} 
CDT1 & 4.3 & 1.0 & 11.2 & CENPN & 3.5 & 1.0 & 8.8
\end{tabular}

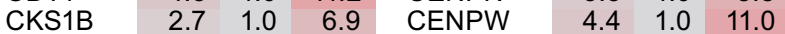

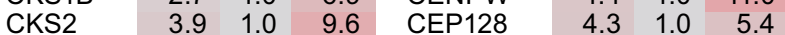
\begin{tabular}{l|l|l|l|l|l|l|l|} 
CLDN 11 & 5.4 & 1.0 & 6.5 & CEP70 & 4.5 & 1.0 & 3.5 \\
\hline
\end{tabular}

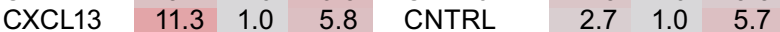
\begin{tabular}{l|rrr|rrrr} 
DBF4 & 4.0 & 1.0 & 6.5 & NCAPG & 9.6 & 1.0 & 12.1
\end{tabular}

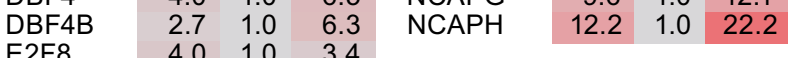
\begin{tabular}{lrrr} 
ESCO2 & 8.4 & 1.0 & 3.4 \\
\hline
\end{tabular} $\begin{array}{lrrrrrrr}\text { ESPL1 } & 7.3 & 1.0 & 2.5 & \text { IL1R1 } & 4.3 & 1.0 & 4.0\end{array}$ $\begin{array}{llllllll}\text { GAS2L3 } & 6.3 & 1.0 & 6.0 & \text { LINC707 } & 4.0 & 1.0 & 43.2 \\ \text { GINS4 } & 2.7 & 1.0 & 4.7 & \text { LMNB1 } & 9.2 & 1.0 & 17.7\end{array}$

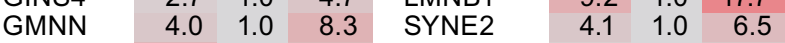

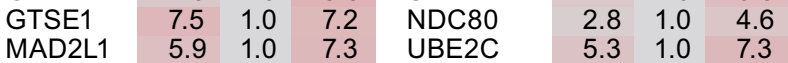
\begin{tabular}{|l|l|l|l|l|l|l|l|} 
MIS18BP1 & 6.6 & 1.0 & 9.3 & UBE2L6 & 3.3 & 1.0 & 3.2 \\
\hline
\end{tabular} \begin{tabular}{lll|l|l} 
MND1 & 5.5 & 1.0 & 13.2 &
\end{tabular} NUSAP1 $\quad 9.7 \quad 1.0 \quad 15.0$ $\begin{array}{llll}\text { ORC1 } & 5.9 & 1.0 & 10.5\end{array}$ $\begin{array}{lrrr}\text { PLK1 } & 6.7 & 1.0 & 9.1 \\ \text { PLK4 } & 9.6 & 1.0 & 12.1\end{array}$ $\begin{array}{lrrr}\text { PLK4 } & 9.6 & 1.0 & 12.1 \\ \text { POLD3 } & 2.0 & 1.0 & 2.9\end{array}$ $\begin{array}{llll}\text { RFC3 } & 3.1 & 1.0 & 3.9\end{array}$ $\begin{array}{llll}\text { RFC5 } & 1.7 & 1.0 & 2.4\end{array}$ $\begin{array}{llll}\text { RRM2 } & 5.1 & 1.0 & 9.4\end{array}$ $\begin{array}{llrr}\text { SKA1 } & 6.4 & 1.0 & 10.7\end{array}$ \begin{tabular}{l|l|l|l|l} 
SMC4 & 7.4 & 1.0 & 8.0
\end{tabular} $\begin{array}{lrrr}\text { TOP2A } & 12.1 & 1.0 & 11.3\end{array}$ TOX TPX2

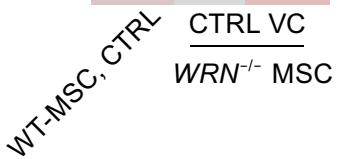

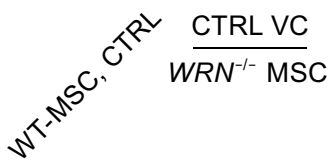


4 Figure 4. VC induced a global transcriptome change of aging-suppressing genes and pathways. (A) Volcano plot showing significantly altered genes $\left(\| \log _{2}\right.$ (Fold change $) \mid>1$, $P$ value $<0.05)$ between vehicle- and VC-treated $W R N^{/-}$MSCs. FC, fold change. (B) KEGG based pathway enrichment analysis of the significantly altered gene sets (green: down regulated genes; red: up regulated genes) in $W R N^{\prime-}$ MSCs upon VC supplement. Number of altered genes in each pathway was indicated by size of the bubble. (C) Predicted protein-protein interactions (PPI) analysis of differentially expressed genes was based on the STRING database, according to e-value $=1 \times$ $10^{-10}$ and string score $>700$, differentially expressing genes with interaction frequency $>80$ were illustrated on the picture. Node size indicated the degree of interaction and node color indicated clustering co-efficiency (green: down regulated genes; red: up regulated genes). (D) Heatmap of mRNA levels between vehicle- and VC-treated WRN ${ }^{\prime-}$ MSCs.

of IL-6, a phenotype of SASP, in the medium of WS MSC cultures (Zhang et al., 2015). We also identify that VC decreases the IL-6 secretion in WS MSCs. This suggests a possibility of employing $\mathrm{VC}$ as a beneficial factor to decrease the inflammatory mediators for WS patients. Given that VC restores the in vivo viability of WS MSC, one may expect that VC could help to eliminate the harmful effects of senescent cells and/or counteract the premature stem cell deterioration in vivo (Baker et al., 2016; Baker et al., 2011). Although human unlike rodents cannot synthesize VC in the body, the water-soluble property of VC makes it very easy to be supplied through dietary sources and supplements. Our study shows that high concentrations of VC (i.e. $280 \mu \mathrm{mol} / \mathrm{L}$ ) produce the best effect on rejuvenating WS MSCs without discernible cytotoxicity (Du et al., 2012). Oral supplement of VC normally reaches micromolar level in the plasma, with the highest plasma concentration around $80 \mu \mathrm{mol} / \mathrm{L}$, which is the suboptimal dose for alleviating the aging process. However, in clinical trials of cancer therapy, intravenous injection of $\mathrm{VC}$ can increase the plasma level of VC up to millimolar levels (Du et al., 2012; Fukushima and Yamazaki, 2010). Thus, it would be of particular interest to titrate the optimal dosage of VC used in clinics from which WS patients will benefit mostly. One concern is that higher level of VC tends to induce free radicals, a phenotypic switch from anti-oxidant to oxidizing agent (Du et al., 2012). Thus the titration for optimal VC concentration in human patients needs to be very cautiously performed.

Regarding the molecular mechanism, we found that treatment with VC effectively diminishes cellular ROS in WS MSCs. This could be one of the molecular mechanisms underlying VC's effect, given that WS MSCs exhibit much higher ROS levels than their wild-type counterparts. We found that, besides VC, other ROS eliminators also show an effect on the rejuvenation of WS MSCs. For example, NAC, a strong antioxidant, and Met and EGCG, activators of NRF2 antioxidant pathway (Martin-Montalvo et al., 2013; Na et al.,
2008), all alleviated the aging defects of WS MSCs. One would postulate that an increased anti-oxidizing capability in WS MSCs could principally counteract the harmful effect of ROS on compromising the biomacromolecule machinery (i.e. DNA or protein) inside the cells. On the other hand, elevated ROS levels have recently been known as a factor leading to heterochromatin disorganization (Frost et al., 2014). In this context, reduction of ROS levels may provide an explanation for restoration of heterochromatin architecture by VC. However, it should also be noted that NAC, a much stronger antioxidant, only show mild rescue activity towards WS MSCs. Therefore, this raises a possibility that VC may also exert an effect in a ROS-scavenger independent manner. Recent evidence points to a more direct role of $\mathrm{VC}$ in regulating epigenetic reprogramming (Young et al., 2015). For instance, VC functions as a cofactor for TET dioxygenases that catalyze the oxidation of 5-methylcytosine $(5 \mathrm{mC})$ to 5 -hydroxymethylcytosine $(5 \mathrm{hmC})$, further resulting in the generation of 5-formylcytosine $(5 \mathrm{fC}), 5$-carboxylcytosine $(5 \mathrm{caC})$, and unmodified cytosine. In addition, VC serves as a cofactor of the JumonjiC (JmjC)-domain containing histone demethylases (i.e. JHDM1) (Chen et al., 2013; Esteban and Pei, 2012; Esteban et al., 2010; Pera, 2013; Wang et al., 2011; Yulin et al., 2012). A more direct evidence is that $\mathrm{VC}$ promotes proliferation of bone marrowderived MSCs and facilitates the derivation of iPSCs from MSCs (Yulin et al., 2012). How VC coordinates different mechanisms in alleviating aging defects in WS MSCs warrants further investigation.

In addition, consistent with a role of $\mathrm{VC}$ in rejuvenating the nuclear lamina and heterochromatin in WS MSCs, our study revealed that $\mathrm{VC}$ reset the gene expression profiles of WS MSCs to a younger state. Our recent study has indicated that a group of genes linked to chromatin condensation, cell cycle, DNA replication, and DNA repair were all repressed in WS MSCs (Zhang et al., 2015). Intriguingly, parts of these genes were reactivated by VC treatment in WS MSCs. These observations verified that VC has the capability to help WS MSCs to reprogram the senescent transcriptome probably by reactivating the stemness-associated core transcriptional network. Moreover, our study also demonstrate that VC treatment can downregulate pro-senescence pathways, including P53, FoXO, and HIF etc., which may provide another layer of mechanisms in recovering young MSC phenotypes (Fig. 5).

\section{MATERIALS AND METHODS}

Cell lines generation, culture and treatment

WRN gene knockout human ESC clones (referred to as $W R N^{1-}$ ESC) were generated as previously described (Zhang et al., 2015). Differentiation, purification, culture, and characterization of WT-ESC and $W R N^{-1-}$-ESC derived mesenchymal stem cells (MSCs) were performed as described previously (Zhang et al., 2015).

Procedure of cell treatments with chemicals at indicated concentrations was illustrated in Fig. 1A, including Vitamin C (VC, 


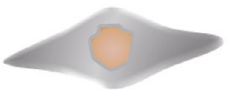

WS-MSC

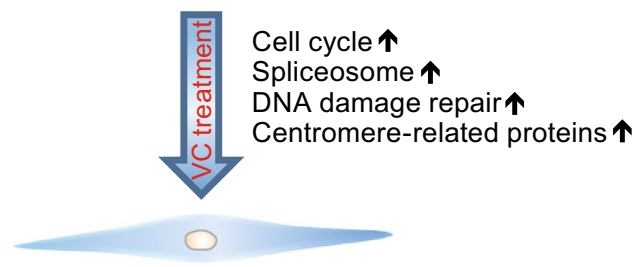

1. Reactivated proliferative potential

2. Repressed expression of $\mathrm{p} 16^{\operatorname{lnk} 4 a}$ and GATA4

3.Alleviated telomere attrition

4.Decreased senescence-associated secretory phenotype (SASP)

5. Rejuvenated heterochromatin and nuclear lamina architecture 6 . Restored the in vivo viability

Figure 5. A proposed model illustrating that VC represses aging properties in WS MSCs by causing a global transcriptome change of aging-suppressing genes and pathways.

Sigma, A4403), (-)-epigallocatechin gallate (EGCG, Sigma, E4143), Vitamin E (VE, Sigma, T1782), N-Actyl-L-cysteine (NAC, Sigma, A7250), Metformin (Met, Tocris, 2864), Rapamycin (Rap, Tocris, 1292), and Resveratrol (Res, Sigma, R5010). VC and NAC were dissolved in water. All other compounds were dissolved in DMSO. Final concentrations of DMSO did not exceed $0.1 \%$.

\section{Reagents}

Antibodies were purchased from the following companies. Abcam: anti-H3K9me3 (ab8898); Santa Cruz Biotechnology: anti$\beta$-actin (SC-130301), anti-GATA4 (SC-1237); Cell Signaling Technology: Phospho-(Ser/Thr) ATM/ATR Substrate Antibody (2851), anti-HP1a (2616), anti-p16 (4828); Millipore: anti-y-H2AX (05-636); BD Bioscience: anti-LAP2 $\beta$ (611000); Vector: anti-Ki67 (VP-RM04).

\section{Senescence associated $\beta$-galactosidase (SA- $\beta$-gal) staining}

SA- $\beta$-gal staining was performed as described previously (DebacqChainiaux et al., 2009). Briefly, cultured cells were washed in PBS and fixed at room temperature for $3 \mathrm{~min}$ in $2 \%$ formaldehyde and $0.2 \%$ glutaraldehyde. Fixed cells were stained with fresh staining solution for SA- $\beta$-gal activity at $37^{\circ} \mathrm{C}$ overnight, and counted for positivity $(\mathrm{N}>300)$.

\section{ROS detection}

Oxidative stress levels were quantified through H2-DCFDA (Invitrogen, C6827) based flow cytometry following manufacturer's instructions

\section{ELISA}

Kits to detect IL-6 (D6050) and IL-8 (D8000C) were purchased from R\&D Systems, and used as previously reported (Zhang et al., 2015). All ELISA data were normalized to cell numbers
Population doubling time (PDT)

Cell numbers of each passage were counted by hemocytometer after trypan blue staining, and then PDT was calculated according to previous formulae (Zhang et al., 2015)

Western bloting and quantitative RT-PCR

For Western blot, preparation of lysate from cell culture was described as previously reported. BCA kit purchased from Thermo Fisher Scientific was used for protein quantification. Equal amounts of protein lysates were subjected to the wells of the SDS-PAGE gel, and then electrotransferred to a PVDF membrane. After blocking, the membrane was incubated with appropriate dilutions of primary antibody and secondary antibodies (Jackson ImmunoResearch Labs). Then imaging was performed using ChemiDoc XRS system (Bio-Rad).

For quantitative analysis of gene expression, total RNA was extracted according to previous protocol (Zhang et al., 2015). Then cDNA was synthesized by GoScript ${ }^{\mathrm{TM}}$ Reverse Transcription System (Promega), followed by removal of genomic DNA with DNAfree $^{\mathrm{TM}}$ Kit from Ambion. Quantitative RT-PCR was performed using SYBR $®$ Green Supermix (TOYOBO). Quantitative PCR-based method was used to measure telomere length according to established protocol (Zhang et al., 2015). Primer sequences are listed in Table S1.

\section{Immunofluorescence microscopy}

Cells were washed once with PBS and fixed in $4 \%$ formaldehyde at room temperature. Subsequently, cells were blocked with $10 \%$ donkey serum and $0.1 \%$ Triton $\mathrm{X}-100$ in PBS for $1 \mathrm{~h}$, and then diluted primary antibodies were added and incubated at $4^{\circ} \mathrm{C}$ overnight. After three consecutive washes in wash buffer, cells were incubated for 45 min with secondary antibodies (Alexa Fluor Donkey-anti-mouse and Alexa Fluor Donkey-anti-rabbit, Invitrogen) together with Hoechst 33342 (Invitrogen). After washed, samples were covered with VECTASHIELD mounting medium (Vector) and imaged in Leica SP5 confocal. Acquisition parameter was same for each experiment. Around 100 randomly selected cells were analyzed.

In vivo cell viability analysis

MSC implantation was performed as previously described (Zhang et al., 2015). In brief, $5 \times 10^{5}$ luciferase-expressing WS MSCs were pretreated with vehicle or $280 \mu \mathrm{mol} / \mathrm{L}$ VC for one week and then implanted into the middle of the tibialis anterior muscle of immunodeficient mice. Seven days after implantation, mice were anaesthetized and injected with D-luciferin solution. Fifteen minutes later, in vivo luciferase activity of each mouse was determined by the IVIS lumina system (PerkinElmer). Luminescence intensity was normalized to luciferase intensity of MSCs just before implantation. Animal experiments performed in this study were approved by the Institute of Biophysics, Chinese Academy of Science.

\section{RNA-seq library construction}

Two million cells were applied to extract total RNA as previously described (Zhang et al., 2015). RNA integrity was assessed using the RNA Nano 6000 Assay Kit of the Bioanalyzer 2100 system 
(Agilent Technologies). A total amount of $3 \mu \mathrm{g}$ RNA per sample was used as input material. For the RNA sample preparations, sequencing libraries were generated using NEBNext ${ }^{\circledR}$ UItra ${ }^{\text {TM }}$ RNA Library Prep Kit for Illumina ${ }^{\circledR}$ (NEB) following manufacturer's recommendations and index codes were added to attribute sequences to each sample. Then, PCR products were purified (AMPure XP system) and library quality was assessed using the Agilent Bioanalyzer 2100 system. At last, the library preparations were sequenced on an Illumina Hiseq platform and 125 bp/150 bp paired-end reads were generated.

\section{RNA-seq data analysis}

Then reads were mapped to the human reference genome hg19 (from UCSC) by TopHat v2.0.12. Transcript expression and differentially expressed genes were analyzed as previously reported $\mathrm{Wu}$ et al., 2015). Briefly, HTSeq v0.6.1 was used to count the reads numbers mapped to each gene. And then FPKM (Fragments per kilobase of transcript sequence per millions) of each gene was calculated based on the length of the gene and reads count mapped to this gene. Differential expression analysis of two groups (two biological replicates per condition) was performed using the DESeq $R$ package (1.18.0). Gene Ontology (GO) enrichment analysis of differentially expressed genes was implemented by the GOseq $R$ package, in which gene length bias was corrected. KOBAS software was used to test the statistical enrichment of differential expression genes in KEGG pathways. GO terms and pathway enrichment with corrected $P$ value less than 0.05 were considered significantly enriched by differential expressed genes. Protein-protein interactions (PPI) prediction of differentially expressed genes was based on the STRING database.

\section{ACKNOWLEDGMENTS}

We thank all lab members for providing helpful feedbacks. This work was supported by the National Basic Research Program of China (973 Program) (Nos. 2015CB964800 and 2014CB910503), the Strategic Priority Research Program of the Chinese Academy of Sciences (XDA01020312), the National High Technology Research and Development Program of China (2015AA020307), the National Natural Science Foundation of China (Grant Nos. 81330008, 31222039, 31201111, 81371342, 81300261, 81300677, 81271266, 81471414, 81422017, and 81401159), the Program of Beijing Municipal Science and Technology Commission (Z151100003915072), the Beijing Natural Science Foundation (7141005 and 5142016), the Key Research Program of the Chinese Academy of Sciences (KJZDEW-TZ-L05), the Thousand Young Talents program of China, Youth Innovation Promotion Association of CAS. WZ was supported by $\mathrm{NIH}$ grants CA158055, CA200673, and CA203834, the V Scholar award, Breast Cancer Research Award and Oberley Award (National Cancer Institute Award P30CA086862) from Holden Comprehensive Cancer Center at the University of lowa, and startup fund from the Department of Pathology, University of lowa.

\section{ABBREVIATIONS}

DDR, DNA damage response; EGCG, (-)-epigallocatechin gallate; ER, endoplasmic reticulum; FPKM, fragments per kilobase of transcript sequence per millions; GO, Gene Ontology; HGPS, Hutchinson-Gliford progeria syndrome; hPSC, human pluripotent stem cells; iPSCs, induced pluripotent stem cells; Met, Metformin; MSCs, mesenchymal stem cells; NAC, N-Actyl-L-cysteine; PDT, population doubling time; PPI, protein-protein interactions; Rap, Rapamycin; Res, Resveratrol; ROS, reactive oxygen species; SA- $\beta$ gal, senescence-associated- $\beta$-galactosidase; WS, Werner syndrome; VC, Vitamin C; VE, Vitamin E.

\section{COMPLIANCE WITH ETHICS GUIDELINES}

Ying Li, Weizhou Zhang, Liang Chang, Yan Han, Liang Sun, Xiaojun Gong, Hong Tang, Zunpeng Liu, Huichao Deng, Yanxia Ye, Yu Wang, Jian Li, Jie Qiao, Jing Qu, Weiqi Zhang, and Guang-Hui Liu declare that they have no conflict of interest. All institutional and national guidelines for the care and use of laboratory animals were followed.

\section{OPEN ACCESS}

This article is distributed under the terms of the Creative Commons Attribution 4.0 International License (http://creativecommons.org/ licenses/by/4.0/), which permits unrestricted use, distribution, and reproduction in any medium, provided you give appropriate credit to the original author(s) and the source, provide a link to the Creative Commons license, and indicate if changes were made.

\section{REFERENCES}

Baker DJ, Wijshake T, Tchkonia T, LeBrasseur NK, Childs BG, van de Sluis B, Kirkland JL, van Deursen JM (2011) Clearance of p16Ink4a-positive senescent cells delays ageing-associated disorders. Nature 479:232-236

Baker DJ, Childs BG, Durik M, Wijers ME, Sieben CJ, Zhong J, Saltness RA, Jeganathan KB, Verzosa GC, Pezeshki A et al (2016) Naturally occurring p16(Ink4a)-positive cells shorten healthy lifespan. Nature 530:184-189

Baur JA, Pearson KJ, Price NL, Jamieson HA, Lerin C, Kalra A, Prabhu VV, Allard JS, Lopez-Lluch G, Lewis K et al (2006) Resveratrol improves health and survival of mice on a highcalorie diet. Nature 444:337-342

Benayoun BA, Pollina EA, Brunet A (2015) Epigenetic regulation of ageing: linking environmental inputs to genomic stability. Nat Rev Mol Cell Biol 16:593-610

Blondel S, Egesipe AL, Picardi P, Jaskowiak AL, Notarnicola M, Ragot J, Tournois J, Le Corf A, Brinon B, Poydenot P et al (2016) Drug screening on Hutchinson Gilford progeria pluripotent stem cells reveals aminopyrimidines as new modulators of farnesylation. Cell Death Dis 7:e2105

Burtner CR, Kennedy BK (2010) Progeria syndromes and ageing: what is the connection? Nat Rev Mol Cell Biol 11:567-578

Campisi J (2013) Aging, cellular senescence, and cancer. Annu Rev Physiol 75:685-705

Cao K, Graziotto JJ, Blair CD, Mazzulli JR, Erdos MR, Krainc D, Collins FS (2011) Rapamycin reverses cellular phenotypes and 
enhances mutant protein clearance in Hutchinson-Gilford progeria syndrome cells. Sci Transl Med 3:89ra58

Chen J, Guo L, Zhang L, Wu H, Yang J, Liu H, Wang X, Hu X, Gu T, Zhou $Z$ et al (2013) Vitamin C modulates TET1 function during somatic cell reprogramming. Nat Genet 45:1504-1509

Cheung HH, Liu X, Canterel-Thouennon L, Li L, Edmonson C, Rennert OM (2014) Telomerase protects werner syndrome lineage-specific stem cells from premature aging. Stem Cell Reports 2:534-546

Dallaire A, Proulx S, Simard MJ, Lebel M (2014) Expression profile of Caenorhabditis elegans mutant for the Werner syndrome gene ortholog reveals the impact of vitamin $C$ on development to increase life span. BMC Genomics 15:940

Davis T, Kipling D (2006) Werner Syndrome as an example of inflamm-aging: possible therapeutic opportunities for a progeroid syndrome? Rejuvenation Res 9:402-407

Debacq-Chainiaux F, Erusalimsky JD, Campisi J, Toussaint O (2009) Protocols to detect senescence-associated beta-galactosidase (SA-betagal) activity, a biomarker of senescent cells in culture and in vivo. Nat Protoc 4:1798-1806

Du J, Cullen JJ, Buettner GR (2012) Ascorbic acid: chemistry, biology and the treatment of cancer. Biochim Biophys Acta 1826:443-457

Duan S, Yuan G, Liu X, Ren R, Li J, Zhang W, Wu J, Xu X, Fu L, Li Y et al (2015) PTEN deficiency reprogrammes human neural stem cells towards a glioblastoma stem cell-like phenotype. Nat Commun 6:10068

Esteban MA, Pei D (2012) Vitamin C improves the quality of somatic cell reprogramming. Nat Genet 44:366-367

Esteban MA, Wang T, Qin B, Yang J, Qin D, Cai J, Li W, Weng Z, Chen J, Ni S et al (2010) Vitamin C enhances the generation of mouse and human induced pluripotent stem cells. Cell Stem Cell 6:71-79

Frost B, Hemberg M, Lewis J, Feany MB (2014) Tau promotes neurodegeneration through global chromatin relaxation. Nat Neurosci 17:357-366

Fu L, Xu X, Ren R, Wu J, Zhang W, Yang J, Ren X, Wang S, Zhao Y, Sun $L$ et al (2016) Modeling xeroderma pigmentosum associated neurological pathologies with patients-derived iPSCs. Protein Cell 7(3):210-221

Fukushima R, Yamazaki E (2010) Vitamin C requirement in surgical patients. Curr Opin Clin Nutr Metab Care 13:669-676

Harrison DE, Strong R, Sharp ZD, Nelson JF, Astle CM, Flurkey K, Nadon NL, Wilkinson JE, Frenkel K, Carter CS et al (2009) Rapamycin fed late in life extends lifespan in genetically heterogeneous mice. Nature 460:392-395

Kang C, Xu Q, Martin TD, Li MZ, Demaria M, Aron L, Lu T, Yankner BA, Campisi J, Elledge SJ (2015) The DNA damage response induces inflammation and senescence by inhibiting autophagy of GATA4. Science 349:aaa5612

Kudlow BA, Kennedy BK, Monnat RJ Jr (2007) Werner and Hutchinson-Gilford progeria syndromes: mechanistic basis of human progeroid diseases. Nat Rev Mol Cell Biol 8:394-404

La Fata G, Weber P, Mohajeri MH (2014) Effects of vitamin E on cognitive performance during ageing and in Alzheimer's disease. Nutrients 6:5453-5472
Labbe A, Turaga RV, Paquet ER, Garand C, Lebel M (2010) Expression profiling of mouse embryonic fibroblasts with a deletion in the helicase domain of the Werner Syndrome gene homologue treated with hydrogen peroxide. BMC Genomics $11: 127$

Lebel M, Massip L, Garand C, Thorin E (2010) Ascorbate improves metabolic abnormalities in Wrn mutant mice but not the free radical scavenger catechin. Ann N Y Acad Sci 1197:40-44

Liu GH, Barkho BZ, Ruiz S, Diep D, Qu J, Yang SL, Panopoulos AD, Suzuki K, Kurian L, Walsh C et al (2011) Recapitulation of premature ageing with iPSCs from Hutchinson-Gilford progeria syndrome. Nature 472:221-225

Liu GH, Ding Z, Izpisua Belmonte JC (2012a) iPSC technology to study human aging and aging-related disorders. Curr Opin Cell Biol 24:765-774

Liu GH, Qu J, Suzuki K, Nivet E, Li M, Montserrat N, Yi F, Xu X, Ruiz S, Zhang $W$ et al (2012b) Progressive degeneration of human neural stem cells caused by pathogenic LRRK2. Nature 491:603-607

Liu GH, Suzuki K, Li M, Qu J, Montserrat N, Tarantino C, Gu Y, Yi F, Xu X, Zhang W et al (2014) Modelling Fanconi anemia pathogenesis and therapeutics using integration-free patient-derived iPSCs. Nat Commun 5:4330

Lo Cicero A, Nissan X (2015) Pluripotent stem cells to model Hutchinson-Gilford progeria syndrome (HGPS): current trends and future perspectives for drug discovery. Ageing Res Rev 24:343-348

Lopez-Otin C, Blasco MA, Partridge L, Serrano M, Kroemer G (2013) The hallmarks of aging. Cell 153:1194-1217

Martin-Montalvo A, Mercken EM, Mitchell SJ, Palacios HH, Mote PL, Scheibye-Knudsen M, Gomes AP, Ward TM, Minor RK, Blouin MJ et al (2013) Metformin improves healthspan and lifespan in mice. Nat Commun 4:2192

Martins R, Lithgow GJ, Link W (2015) Long live FOXO: unraveling the role of FOXO proteins in aging and longevity. Aging Cell 15 (2):196-207

Miller JD, Ganat YM, Kishinevsky S, Bowman RL, Liu B, Tu EY, Mandal PK, Vera E, Shim JW, Kriks S et al (2013) Human iPSCbased modeling of late-onset disease via progerin-induced aging. Cell Stem Cell 13:691-705

Na HK, Kim EH, Jung JH, Lee HH, Hyun JW, Surh YJ (2008) (-)Epigallocatechin gallate induces Nrf2-mediated antioxidant enzyme expression via activation of PI3K and ERK in human mammary epithelial cells. Arch Biochem Biophys 476:171-177

Pan H, Guan D, Liu X, Li J, Wang L, Wu J, Zhou J, Zhang W, Ren R, $\mathrm{Li} Y$ et al (2016) SIRT6 safeguards human mesenchymal stem cells from oxidative stress by coactivating NRF2. Cell Res 26:190-205

Park IH, Arora N, Huo H, Maherali N, Ahfeldt T, Shimamura A, Lensch MW, Cowan C, Hochedlinger K, Daley GQ (2008) Disease-specific induced pluripotent stem cells. Cell 134:877-886

Pera MF (2013) Epigenetics, vitamin supplements and cellular reprogramming. Nat Genet 45:1412-1413

Polosak J, Kurylowicz A, Roszkowska-Gancarz M, Owczarz M, Puzianowska-Kuznicka M (2011) Aging is accompanied by a progressive decrease of expression of the WRN gene in human blood mononuclear cells. J Gerontol A Biol Sci Med Sci 66:19-25 
Saha K, Jaenisch R (2009) Technical challenges in using human induced pluripotent stem cells to model disease. Cell Stem Cell 5:584-595

Wang T, Chen K, Zeng X, Yang J, Wu Y, Shi X, Qin B, Zeng L, Esteban MA, Pan G et al (2011) The histone demethylases Jhdm1a/1b enhance somatic cell reprogramming in a vitamin-Cdependent manner. Cell Stem Cell 9:575-587

Wu H, Wei L, Fan F, Ji S, Zhang S, Geng J, Hong L, Fan X, Chen Q, Tian $J$ et al (2015) Integration of Hippo signalling and the unfolded protein response to restrain liver overgrowth and tumorigenesis. Nat Commun 6:6239

Yang J, Cai N, Yi F, Liu GH, Qu J, Izpisua Belmonte JC (2014) Gating pluripotency via nuclear pores. Trends Mol Med 20:1-7

Young JI, Zuchner S, Wang G (2015) Regulation of the Epigenome by Vitamin C. Annu Rev Nutr 35:545-564
Yu DX, Marchetto MC, Gage FH (2013) Therapeutic translation of iPSCs for treating neurological disease. Cell Stem Cell 12:678688

Yulin X, Lizhen L, Lifei Z, Shan F, Ru L, Kaimin H, Huang H (2012) Efficient generation of induced pluripotent stem cells from human bone marrow mesenchymal stem cells. Folia Biol (Praha) 58:221-230

Zhang W, Qu J, Suzuki K, Liu GH, Izpisua Belmonte JC (2013) Concealing cellular defects in pluripotent stem cells. Trends Cell Biol 23:587-592

Zhang W, Li J, Suzuki K, Qu J, Wang P, Zhou J, Liu X, Ren R, Xu X, Ocampo A et al (2015) Aging stem cells. A Werner syndrome stem cell model unveils heterochromatin alterations as a driver of human aging. Science 348:1160-1163 